УДК 514.112.3

\title{
On Triangles with Sides That Form an Arithmetic Progression
}

\author{
Yu.N. Maltsev, A.S. Monastyreva
}

Altai State Pedagogical University (Barnaul, Russia)

\section{О треугольниках, стороны которых образуют арифметическую прогрессию}

\author{
Ю.Н. Мальцев, А.С. Монастьрева
}

Алтайский государственный педагогический университет

(Барнаул, Россия)

Properties of triangles such that the squares of their sides form an arithmetic progression were studied in 2018. In this paper, triangles with sides that form an arithmetic progression are described. Let a, b, c be sides of an arbitrary triangle $\mathrm{ABC}$. If sides $\mathrm{b}, \mathrm{a}, \mathrm{c}$ of the triangle $\mathrm{ABC}$ form an arithmetic progression then, for example, the equality $a=(b+c) / 2(b<a<c)$ holds. The class of triangles for which $a=(b+c) / 2$ is greater than the class of triangles for which b, a, c form an arithmetic progression. In this paper, we study the properties of triangles for which this equality holds. Thus, triangles with sides that form an arithmetic progression are described with the help of the parameters $\mathrm{p}, \mathrm{R}, \mathrm{r}$. Classes of rectangular triangles, triangles with angle $30^{\circ}$, triangles with angle $60^{\circ}$, triangles with angle $120^{\circ}$ are studied and described.

Key words: triangle, circumradius, inradius, semiperimeter, arithmetic progression.

\section{DOI 10.14258/izvasu(2020)1-18}

1. Introduction. Let $R$ and $r$ be the circumradius and the inradius of an arbitrary triangle $A B C$. Also, let $A B=c, A C=b, B C=a$, $\angle A=\alpha, \angle B=\beta, \angle C=\gamma, p=\frac{a+b+c}{2}$.

In [1], the authors studied properties of triangles such that the squares of its sides form an arithmetic progression. Also, description of such triangles associated with its remarkable points is given. It is natural to describe triangles such that its sides form an arithmetic progression. In this case, for examples, the equality $a=\frac{b+c}{2}(b<a<c)$ holds. The class
В 2018 г. описаны свойства треугольников, у которых квадраты сторон образуют арифметическую прогрессию. В данной работе описываются треугольники, стороны которых образуют арифметическую прогрессию. Пусть a, b, с - это стороны произвольного треугольника АВС. Если стороны b, a, с треугольника АВC образуют арифметическую прогрессию, то, например, следующее равенство выполняется: $\mathrm{a}=(\mathrm{b}+\mathrm{c}) / 2(\mathrm{~b}<\mathrm{a}<\mathrm{c})$. Однако класс треугольников, для которых равенство $\mathrm{a}=(\mathrm{b}+\mathrm{c}) / 2$ выполняется, больше, чем класс треугольников, стороны которых b, a, с образуют арифметическую прогрессию. В настоящей работе мы изучаем свойства треугольника, для сторон которого выполняется это равенство. И это позволяет описать (на языке параметров p, R, r) треугольники, стороны которых образуют арифметическую прогрессию. Отдельно описываются классы треугольников: прямоугольные треугольники, треугольники с углом $30^{\circ}$, треугольники с углом $60^{\circ}$, треугольники с углом $120^{\circ}$.

Ключевые слова: треугольник, радиус описанной окружности, радиус вписанной окружности, полупериметр, арифметическая прогрессия.

of triangles for which $a=\frac{b+c}{2}$ is greater than the class of triangles for which $b, a, c$ form an arithmetic progression. Really, the class of triangles for which $a=\frac{b+c}{2}$ also contains all equilateral triangles. Further, this class contains rectangular triangle with sides 3, 4, 5. In [2, Ex. 352], [3, Ex. 286, 287, 321], [4, Ex. 11.55, 12.23], [5, p. 89, Ex. 38], and [6, Ex. 88 ], some properties and characterizations of such triangles are given.

In this paper, study of this triangles is continued and the following theorems are proved [7]. 
Theorem 1. For arbitrary triangle $A B C$, the following conditions are equivalent:

1. $a=\frac{b+c}{2}$;

2. $p^{2}=18 R r-9 r^{2}$;

3. the sides of the triangle $A B C$ are equal to $\frac{2 p}{3}-\sqrt{2 r(R-2 r)}, \frac{2 p}{3}, \frac{2 p}{3}+\sqrt{2 r(R-2 r)}$.

Besides this, $\alpha \leq 60^{\circ}$.

Theorem 2. Let $R, r$ be arbitrary positive numbers such that $R \geq 2 r$ and $p=\sqrt{18 R r-9 r^{2}}$. Then there exists a unique triangle $A B C$ such that $R, r, p$ are the circumradius, the inradius, the semiperimeter of the triangle $A B C$ respectively.

Theorem 3. (1) Let $\triangle A B C$ be a rectangular triangle. The equality $a=\frac{b+c}{2}$ holds in $\triangle A B C$ iff $p=6 r, R=\frac{5}{2} r$ (i.e. in this case, $\triangle A B C$ is homothetic to a triangle with sides $3,4,5)$.

(2) Let $\triangle A B C$ be a triangle with angle $60^{\circ}$. The equality $a=\frac{b+c}{2}$ holds in $\triangle A B C$ iff $\triangle A B C$ is a equilateral triangle.

(3) Let $\triangle A B C$ be a triangle with angle $120^{\circ}$. The equality $a=\frac{b+c}{2}$ holds in $\triangle A B C$ iff $p=5 \sqrt{3} r$, $R=\frac{14}{3} r$ (i.e. in this case, $\triangle A B C$ is homothetic to a triangle with sides $6,10,14)$.

\section{Proof of the main results.}

Proof of Theorem 1. Let $\triangle A B C$ be an arbitrary triangle such that $a=\frac{b+c}{2}$. Then $a=\frac{2 p}{3}$. By [8], $a, b, c$ are roots of the equation

$$
x^{3}-2 p x^{2}+\left(p^{2}+r^{2}+4 R r\right) x-4 p R r=0 .
$$

Therefore

$$
\left(\frac{2 p}{3}\right)^{3}-2 p\left(\frac{2 p}{3}\right)^{2}+\left(p^{2}+r^{2}+4 R r\right) \cdot \frac{2 p}{3}-4 p R r=0
$$

and $p^{2}=18 R r-9 r^{2}$. So (1) implies (2).

Now prove that (2) implies (1). Assume that $p^{2}=$ $18 R r-9 r^{2}$. Then $\frac{2 p}{3}$ is a root of the equation (1) and

$$
\begin{aligned}
x^{3}-2 p x^{2}+ & \left(p^{2}+r^{2}+4 R r\right) x-4 p R r= \\
& =\left(x-\frac{2 p}{3}\right)\left(x^{2}-\frac{4 p}{3} x+6 R r\right) .
\end{aligned}
$$

It implies that the numbers

$$
\begin{aligned}
b=\frac{2 p}{3}-\sqrt{2 r(R-2 r)}, a & =\frac{2 p}{3}, \\
c & =\frac{2 p}{3}+\sqrt{2 r(R-2 r)}
\end{aligned}
$$

are roots of (1) and the equality $a=\frac{2 p}{3}=\frac{b+c}{2}$ holds in $\triangle A B C$. Note that we have proved also that the condition ( 1 ) is equivalent to the condition (3). We will show $\alpha=\angle B A C \leq 60^{\circ}$. We need to prove the following lemma.

Lemma. $\cos \alpha=1-\frac{r}{R}$.

Proof. The equality $a=\frac{b+c}{2}$ is equivalent to $a^{2}=(b+c-a)^{2}$. In its turn, the condition $a^{2}=$ $(b+c-a)^{2}$ is equivalent to the equality

$$
\begin{aligned}
1- & \frac{b^{2}+c^{2}-a^{2}}{2 b c}= \\
& =2\left(1-\frac{a^{2}+c^{2}-b^{2}}{2 a c}\right)\left(1-\frac{a^{2}+b^{2}-c^{2}}{2 a b}\right)
\end{aligned}
$$

and hence

$$
\frac{1-\cos \alpha}{2}=4 \cdot \frac{1-\cos \beta}{2} \cdot \frac{1-\cos \gamma}{2} .
$$

The last equality can be written as $\sin ^{2} \frac{\alpha}{2}=$ $4 \sin ^{2} \frac{\beta}{2} \sin ^{2} \frac{\gamma}{2}$, i.e. $\sin \frac{\alpha}{2}=2 \sin \frac{\beta}{2} \sin \frac{\gamma}{2}$. By [8], $\sin \frac{\alpha}{2} \sin \frac{\beta}{2} \sin \frac{\gamma}{2}=\frac{r}{4 R}$. Thus

$$
\sin ^{2} \frac{\alpha}{2}=2 \sin \frac{\alpha}{2} \sin \frac{\beta}{2} \sin \frac{\gamma}{2}=\frac{r}{2 R} .
$$

Hence $\cos \alpha=1-2 \sin ^{2} \frac{\alpha}{2}=1-\frac{r}{R}$ and the lemma is proved.

It is known that $R \geq 2 r$ (see [8-10]). Therefore $\cos \alpha=1-\frac{r}{R} \geq \frac{1}{2}$ and $\alpha \leq 60^{\circ}$. The proof is complete.

Proof of Theorem 2. Assume that $R, r$ are arbitrary positive numbers such that $R \geq 2 r$ and $p=\sqrt{18 R r-9 r^{2}}$. By [8, Theorem 2, P. 54], positive numbers $R, r, p$ are the circumradius, the inradius, the semiperimeter of some triangle respectively iff the condition

$$
\left(p^{2}-2 R^{2}-10 R r+r^{2}\right)^{2} \leq 4 R(R-2 r)^{3}
$$

holds. Note that $p=\sqrt{18 R r-9 r^{2}}$ implies (2) Really, we have

$\left(p^{2}-2 R^{2}-10 R r+r^{2}\right)^{2}=4(R-2 r)^{4} \leq 4 R(R-2 r)^{3}$.

The proof is complete.

Proof of Theorem 3. Prove (1). Consider a rectangular triangle $\triangle A B C$ in which $\gamma=90^{\circ}$. By [8, P. 26], $\cos \alpha \cos \beta \cos \gamma=\frac{1}{4 R^{2}}\left(p^{2}-(2 R+r)^{2}\right)$. Since $\gamma=\angle A C B=90^{\circ}$, we have $p=2 R+r$. By Theorem 1 , the equality $a=\frac{b+c}{2}$ is equivalent to the condition $p^{2}=18 R r-9 r^{2}=(2 R+r)^{2}$, or $2 R^{2}-$ $7 R r+5 r^{2}=(R-r)(2 R-5 r)=0$. Since $R \geq 2 r$ 
(the Euler's inequality), the equality $a=\frac{b+c}{2}$ is equivalent to $R=\frac{5 r}{2}$ for the rectangular triangle $\triangle A B C$. So $p=2 R+r=6 r, a=\frac{2 p}{3}=4 r, c=$ $2 R=5 r, b=3 r$ for such triangle. Thus $\triangle A B C$ is homothetic to a triangle with sides $3,4,5$.

Further prove (2). Let $\triangle A B C$ be a triangle with angle $60^{\circ}$. By [8], the numbers $\cos \alpha, \cos \beta, \cos \gamma$ are roots of the equation

$$
\begin{aligned}
4 R^{2} x^{3}-4 R(R+r) x^{2} & +\left(p^{2}+r^{2}-4 R^{2}\right) x+ \\
& +(2 R+r)^{2}-p^{2}=0 .
\end{aligned}
$$

Hence $\cos 60^{\circ}=\frac{1}{2}$ is a root of $(3)$ and $p=\sqrt{3}(R+r)$. By Theorem 1, the equality $a=\frac{b+c}{2}$ is equivalent to the condition $p^{2}=18 R r-9 r^{2}$. Since $p^{2}=3\left(R^{2}+\right.$ $\left.2 R r+r^{2}\right)$, we have $3 R^{2}+6 R r+3 r^{2}=18 R r-9 r^{2}$, i.e. $(R-2 r)^{2}=0$. Therefore the equality $a=\frac{b+c}{2}$ is equivalent to $R=2 r$ for any triangle with angle $60^{\circ}$. By [8, P. 8], the last inequality holds if and only if $\triangle A B C$ is an equilateral triangle.

Finally, prove (3). Let $\triangle A B C$ be a triangle with angle $120^{\circ}$. Then $\cos 120^{\circ}=-\frac{1}{2}$ is a root of (3) and $p=\frac{3 R+r}{\sqrt{3}}$. Thus, by Theorem 1 , the equality $a=$ $\frac{b+c}{2}$ is equivalent to the condition $p^{2}=18 R r-$ $9 r^{2}=\frac{9 R^{2}+6 R r+r^{2}}{3}$ or $R=\frac{14}{3} r$. The last equality implies

$$
\begin{gathered}
p=\frac{3 R+r}{\sqrt{3}}=5 \sqrt{3} r, a=\frac{2 p}{3}=\frac{10 \sqrt{3}}{3} r, \\
b=\frac{2 p}{3}-\sqrt{2 r(R-2 r)}=2 \sqrt{3} r \\
\quad c=\frac{2 p}{3}+\sqrt{2 r(R-2 r)}=\frac{14}{\sqrt{3}} r
\end{gathered}
$$

and $\triangle A B C$ is homothetic to a triangle with sides $6,10,14$.

The proof is complete.

Let $A B C$ be a triangle with angle $30^{\circ}$. Then $\cos 30^{\circ}$ is a root of $(3)$, i.e.

$$
\begin{array}{r}
4 R^{2} \cdot \frac{3 \sqrt{3}}{8}-4 R(R+r) \frac{3}{4}+\left(p^{2}+r^{2}-4 R^{2}\right) \cdot \frac{\sqrt{3}}{2}+ \\
+(2 R+r)^{2}-p^{2}=0 \\
R+r(2+\sqrt{3})=p
\end{array}
$$

If $a=\frac{b+c}{2}$, then, by theorem $1, p^{2}=18 R r-9 r^{2}$, i.e. $18 R r-9 r^{2}=R^{2}+2(2+\sqrt{3}) R r+r^{2}(7+4 \sqrt{3})$, or $R^{2}-2 R r(7-\sqrt{3})+r^{2}(16+4 \sqrt{3})=0$. Hence either $R=(4+2 \sqrt{3}) r$, or $R=(10-4 \sqrt{3}) r$, and $p=R+r(2+\sqrt{3})$. Consider these cases.

Case 1. Let $R=(4+2 \sqrt{3}) r$.

In this case, by Theorem 1, the sides of the triangle $A B C$ are equal to

$$
\begin{gathered}
a=\frac{2 p}{3}=\frac{2}{3}(R+r(2+\sqrt{3}))= \\
=\frac{2}{3}(6+3 \sqrt{3}) r=(4+2 \sqrt{3}) r \\
b=\frac{2 p}{3}-\sqrt{2 r(R-2 r)}= \\
=\frac{2}{3}(R+r(2+\sqrt{3}))-\sqrt{2 r(2+2 \sqrt{3}) r}= \\
=(4+2 \sqrt{3}-2 \sqrt{\sqrt{3}+1}) r \\
c=\frac{2 p}{3}+\sqrt{2 r(R-2 r)}=(4+2 \sqrt{3}+2 \sqrt{\sqrt{3}+1}) r
\end{gathered}
$$

and $\triangle A B C$ is homothetic to a triangle with sides

$$
\begin{aligned}
(4+2 \sqrt{3}+2 \sqrt{\sqrt{3}+1}), 4+2 \sqrt{3} & \\
& (4+2 \sqrt{3}-2 \sqrt{\sqrt{3}+1}) .
\end{aligned}
$$

Case 2. Let $R=(10-4 \sqrt{3}) r$.

In this case, the sides and the semiperimeter of the triangle $A B C$ are equal to

$$
\begin{gathered}
a=\frac{2 p}{3}=\frac{2}{3}(12-3 \sqrt{3})=(8-2 \sqrt{3}) r, \\
p=R+r(2+\sqrt{3})=(12-3 \sqrt{3}) r, \\
b=\frac{2 p}{3}-\sqrt{2 r(R-2 r)}=(10-4 \sqrt{3}) r, c=6 r .
\end{gathered}
$$

Then $\triangle A B C$ is homothetic to a triangle with sides $8-2 \sqrt{3}, 10-4 \sqrt{3}, 6$.

So the following theorem is true.

Theorem 4. Let $A B C$ be a triangle with angle $30^{\circ}$ and the equality $a=\frac{b+c}{2}$ holds in $\triangle A B C$. Then either $\triangle A B C$ is homothetic to a triangle with sides $8-2 \sqrt{3}, 10-4 \sqrt{3}, 6$, or $\triangle A B C$ is homothetic to a triangle with sides

$$
\begin{aligned}
(4+2 \sqrt{3}+2 \sqrt{\sqrt{3}+1}), 4+2 \sqrt{3} \\
(4+2 \sqrt{3}-2 \sqrt{\sqrt{3}+1}) .
\end{aligned}
$$


Consider the class $K(\varphi)$ of triangles with fixed angle $\varphi(0<\varphi<\pi)$ such that the equality $a=\frac{b+c}{2}$ holds for sides $a, b, c$ (in particular, if $b, a, c$ form an arithmetic progression). By [8, p. 26],

$$
\begin{aligned}
p^{2}(1- & \cos \varphi)=4 R^{2} \cos ^{3} \varphi-4 R(R+r) \cos ^{2} \varphi+ \\
& +\left(r^{2}-4 R^{2}\right) \cos \varphi+(2 R+r)^{2}= \\
= & R^{2}\left(4 \cos ^{3} \varphi-4 \cos ^{2} \varphi-4 \cos \varphi+4\right)+ \\
& +R r \cdot 4\left(1-\cos ^{2} \varphi\right)+r^{2}(1+\cos \varphi),
\end{aligned}
$$

$p^{2}=R^{2} \cdot 4 \sin ^{2} \varphi+4(1+\cos \varphi) R r+c t g^{2} \frac{\varphi}{2} r^{2}=18 R r-9 r^{2}$.

Let $\lambda=\frac{R}{r} \geq 2$. Then $\lambda$ is a root of

$$
4 \sin ^{2} \varphi \cdot t^{2}+(4 \cos \varphi-14) t+\left(\operatorname{ctg}^{2} \frac{\varphi}{2}+9\right)=0 .
$$

The equation (4) has no more than two different roots $\lambda_{1}, \lambda_{2}$. Let $r$ be a fixed positive number. For each $\lambda_{i}(i=1,2)$, we can calculate $R_{i}=\lambda_{i} r$, $p_{i}=\sqrt{18 R_{i} r-9 r^{2}}=r \sqrt{18 \lambda_{i}-9}, a_{i}=\frac{2 p_{i}}{3}, b_{i}=$ $\frac{2 p_{i}}{3}-\sqrt{2 r\left(R_{i}-2 r\right)}, c_{i}=\frac{2 p_{i}}{3}+\sqrt{2 r\left(R_{i}-2 r\right)}, i \leq 2$. So the class $K(\varphi)$ has no more than two different subclasses of homothetic triangles. For example, we have:

1. the class $K\left(90^{\circ}\right)$ consists of triangles that are homothetic to a triangle with sides $3,4,5$;

2. the class $K\left(30^{\circ}\right)$ consists of triangles that are homothetic to a triangle with sides either $8-2 \sqrt{3}, 6, \quad 10-$ $4 \sqrt{3}, \quad$ or $\quad(4+2 \sqrt{3}+2 \sqrt{\sqrt{3}+1}), 4 \quad+$ $2 \sqrt{3},(4+2 \sqrt{3}-2 \sqrt{\sqrt{3}+1})$;

3. the class $K\left(60^{\circ}\right)$ consists of equilateral triangles;

4. the class $K\left(120^{\circ}\right)$ consists of triangles that are homothetic to a triangle with sides $3,5,7$.

\section{Библиографический список}

1. Bataille M. On the centres of root-meansquare triangles // Crux Mathematicorum. 2018. Vol. 44(2).

2. Lidskii V.B., Ovsyannikov L.V., Tulaykov A.N., Shabunin M.I. Tasks of elementary mathematics. M., 1965 (in Russian).

3. Scopec Z.A., Zharov V.A. Tasks and theorems of geometry. M., 1962 (in Russian).

4. Prasolov V.V. Tasks of plane geometry. M., 1986 (in Russian).

5. Galperin G.A., Tolpygo A.K. Moskow Mathematical Olympiadas. M., 1986 (in Russian).

6. Morozova E.A., Petrakov I.S. Internatiomal Mathematical Olympiadas. M., 1968 (in Russian).
7. Maltsev Yu.N., Monastyreva A.S. On Triangles Such That its Sides Form an Arithmetic Progression // Conference "Mathematics to Altai region"(Russia, Barnaul). 2019.

8. Soltan V., Maidman S. Identities and inequalities in a triangle. Kishinev, 1982 (in Russian).

9. Zetel S.I. New geometry of a triangle. M., 1962 (in Russian).

10. Maltsev Yu.N., Monastyreva A.S. Selected lectures on the geometry of triangle and quadrangle. Barnaul, 2016 (in Russian). 